\title{
Tuberculosis en América Latina y su impacto en pediatría
}

\author{
Tuberculosis in Latin America and its Impact on Pediatrics \\ Kattia Camacho-Badilla, ${ }^{*}$ Elsa Camilo-de Amarante, ${ }^{\ddagger}$ Celia Martínez de Cuellar, ${ }^{\S}$ \\ José Iván Castillo-Bejarano, " Abiel Homero Mascareñas-De Los Santos, " Pío López-López ${ }^{\mathbb{}}$ \\ * Servicio de Infectología, Hospital Nacional de Niños «Dr. Carlos Sáenz Herrera», San José, Costa Rica. \\ ‡ Unidad de Tuberculosis Infantil, Hospital Infantil «Dr. Robert Reid Cabral», Santo Domingo, República Dominicana. \\ § Instituto de Medicina Tropical, Asunción, Paraguay. Cátedra de Pediatría. Facultad de Ciencias Médicas. Universidad Nacional de Asunción, Paraguay. \\ " Departamento de Pediatría, División de Infectología Pediátrica, Hospital Universitario "Dr. José Eleuterio González», Monterrey, Nuevo León, México. \\ " Presidente de la Sociedad Latinoamericana de Infectología Pediátrica, Jefe del Departamento de Pediatría, Hospital Universitario del Valle.
}

RESUMEN

Durante 2018 se registraron 1.45 millones de muertes por tuberculosis (TB), las cuales colocan a la TB como la principal causa de muerte por un solo microorganismo por encima del VIH. El abordaje diagnóstico-terapéutico representa un reto para el clínico, por lo que la presente revisión documenta los aspectos más relevantes del panorama de la tuberculosis en niños.

Palabras clave: Tuberculosis, tuberculosis multidrogorresistente, Mycobacterium tuberculosis.

\section{INTRODUCCIÓN}

Durante 2018 se registraron 1.45 millones de muertes por tuberculosis (TB), las cuales colocan a la TB como la principal causa de muerte por un solo microorganismo por encima del VIH. ${ }^{1}$

La resistencia a fármacos de primera línea tiene un impacto directo en el pronóstico de los pacientes. La monorresistencia a isoniazida (INH-R) se ha asociado a un aumento de fallas terapéuticas, recurrencia y adquisición de resistencia a rifampicina. ${ }^{2,3}$ Actualmente $78 \%$ de los casos de TB resistente a rifampicina presentan resistencia concomitante a isoniazida. La efectividad del tratamiento de estos casos es de $56 \%{ }^{1}$

Financiamiento: Ninguno.

Conflicto de intereses: Ninguno.

https://dx.doi.org/10.35366/94416

Rev Latin Infect Pediatr 2020; 33 (2): 66-73

\section{ABSTRACT}

During 2018, 1.45 million deaths from tuberculosis (TB) were registered, this place TB as the main cause of death by a single microorganism, above HIV. Diagnostic-therapeutic approach represent a challenge for the clinician, the aim of this review is to document the most relevant aspects of the tuberculosis panorama in children.

Keywords: Tuberculosis, multidrugresistant tuberculosis, Mycobacterium tuberculosis.

El abordaje diagnóstico-terapéutico representa un reto para el clínico, por lo que la presente revisión documenta los aspectos más relevantes del panorama de la tuberculosis en niños.

\section{EPIDEMIOLOGÍA}

A pesar de los grandes avances en el control de la TB en los últimos 20 años y de los esfuerzos realizados, la TB sigue constituyendo un importante problema de salud pública que produce sufrimiento humano y pérdidas económicas, principalmente por tener su mayor incidencia en las poblaciones jóvenes más productivas y en los grupos más vulnerables como las poblaciones marginales de ciudades, personas con $\mathrm{VIH}$, indígenas y personas privadas de la libertad (PPL).

La Organización Mundial de la Salud (OMS) estima que a nivel global 10.4 millones de personas desarrollaron TB en 2018, de las cuales 5.7 millones fueron 
Rev Latin Infect Pediatr 2020; 33 (2): 66-73

hombres, 3.2 millones mujeres y 1.1 millones fueron niños, siendo que la mayoría de estos niños nunca serán diagnosticados o tratados por su enfermedad. ${ }^{1} \mathrm{EI}$ mismo año fueron notificados a la OMS siete millones de casos nuevos de TB, lo cual refleja una brecha de tres millones de casos nuevos entre los casos incidentes y los notificados. La tuberculosis es la principal causa de muerte en las personas con $\mathrm{VIH}$ y es una causa importante de muertes relacionadas con la resistencia a los antimicrobianos. En áreas de alta incidencia, la tuberculosis sigue siendo una de las 10 principales causas de muerte en niños pequeños. ${ }^{1}$ Durante el año $2018,1.5$ millones de personas murieron a causa de la TB, incluyendo 251,000 personas con VIH.

En las Américas se estimó un total de 289,000 $(268,000-310,000)$ casos de tuberculosis, con una tasa de incidencia de 29 (de 27 a 31) casos por 100,000 habitantes en el año 2018. De los 289,000 casos, 33,200 casos nuevos correspondían al grupo de menores de 15 años. Ese mismo año, sólo se notificaron 10,292 casos (31\%) en este último grupo de edad. Al analizar esta situación por grupo de edad, se observa que la mayor brecha de detección se encuentra en el grupo de los menores de cinco años, seguido del grupo de niños de cinco a 14 años y del grupo de mayores de 14 años, cuyos casos no fueron diagnosticados en 72,67 y $12 \%$, respectivamente. ${ }^{1,2}$

La información sobre la situación epidemiológica de la TB en el grupo pediátrico es insuficiente, recientemente la OMS comenzó a publicar datos de este grupo. Siguiendo la iniciativa de la OMS, muchos países comenzaron a publicar los resultados de sus programas de TB en la infancia; sin embargo, la información sobre el tema aún es escasa. ${ }^{3}$ Los desafíos como el diagnóstico temprano y/o adecuado, el tratamiento temprano y la notificación oportuna dificultan la publicación de datos precisos. Sumado a esto, el abordaje de la TB en el niño enfrenta numerosos desafíos relacionados con las manifestaciones clínicas inespecíficas, que junto con la presentación de formas paucibacilares, la dificultad para la expectoración en los más pequeños, las pruebas diagnósticas para la confirmación microbiológica menos sensibles que en el adulto, contribuyen al retraso en el diagnóstico en este grupo de pacientes. Esto es particularmente grave, pues los niños pequeños recientemente infectados con Mycobacterium tuberculosis tienen mayor riesgo de desarrollar enfermedad tuberculosa, incluyendo las formas más graves.

La TB en $\leq 14$ años representó $8 \%$ del total de casos reportados en el mundo en el año 2018, siendo su frecuencia variable (de 4 a 13\%) en las diferentes regiones; África (9\%), las Américas (5\%), Región Mediterránea Este (13\%), Europa (4\%), Sudeste de Asia $7 \%$ y Región del Pacífico Oeste $5 \%$. Asimismo, en los países de América la detección de la TB infantil es muy variable.

En el niño la TB extrapulmonar es más frecuente que en el adulto, representa entre 15 y $20 \%$ de todas las formas de presentación. ${ }^{4,5}$ En un estudio publicado por Bezerra Souza y colaboradores en Ceará, Brasil se reportan 88 casos hospitalizados de TB en $\leq 18$ años, y $25 \%$ de los pacientes con TB extrapulmonar. ${ }^{6}$ En Buenos Aires, Argentina la TB extrapulmonar representó $24 \%(382 / 1607)$ de los casos pediátricos atendidos en el Hospital Ricardo Gutiérrez entre 2000 y 2015. ${ }^{7}$ Por su parte, Carreto-Binaghi y su equipo reportaron que $36.4 \%$ de los casos de TB en < 15 años hospitalizados en el INER entre 2015 y 2017 fueron formas extrapulmonares. ${ }^{8}$ En Paraguay, la TB extrapulmonar se observó en $37.8 \%$ de los pacientes $\leq 19$ años hospitalizados en el Instituto de Medicina Tropical de Asunción, Paraguay. ${ }^{9}$ Zabaleta Ay colegas en Colombia reportaron que 25.9\% de los casos de TB sensible fueron extrapulmonares en 565 casos $\leq 15$ años. ${ }^{10}$ Por su parte, Vázquez Rosales y colaboradores en México reportan que la TB-EP representó $69.9 \%$ de los casos hospitalizados en un centro de referencia ${ }^{11}$ (Tabla 1).

\begin{tabular}{lcccc}
\hline & Tabla 1: Frecuencia de la tuberculosis extrapulmonar en niños 2010-2018. \\
\hline \multicolumn{1}{c}{ Publicación } & País & Núm. de casos & TB-EP n (\%) & Años \\
\hline Bezerra Souza et al. & Brasil & 88 & $20(27.4)$ & $2010-2015$ \\
Neyro SN et al. & Argentina & 1,607 & $382(24.0)$ & $2000-2015$ \\
Carreto-Binaghi LE et al. & México & 22 & $8(36.4)$ & $2015-2017$ \\
Martínez de Cuellar C et al. & Paraguay & 98 & $37(37.8)$ & $2011-2018$ \\
Zabaleta A et al. & Colombia & 505 & $131(25)$ & $2010-2015$ \\
Vázquez Rosales JG et al. & México & 93 & $65(69.9)$ & $2010-2013$
\end{tabular}


Rev Latin Infect Pediatr 2020; 33 (2): 66-73

La TB ganglionar representa casi $40 \%$ de todas las formas extrapulmonares, mientras que la TB diseminada (miliar) y la meníngea son más frecuentes en pacientes de menor edad (< tres años) y en pacientes con $\mathrm{VIH} .{ }^{12,13}$ Asimismo, en el estudio realizado por Vázquez-Rosales y su equipo en niños con TB hospitalizados, la TB ganglionar representó $24.7 \%$ (23/93), seguida de la TB miliar en $16.1 \%(15 / 93) .{ }^{11}$ En México, en 22 pacientes hospitalizados la TB ganglionar fue identificada en $18.2 \%$ de los casos (4/22), seguida de la TB pleural en $9.1 \%(2 / 22){ }^{5}$

La información sobre la TB-DR en niños también es insuficiente. Zabaleta $A$ y colaboradores han analizado 565 casos, de los cuales 503 (89.0\%) eran nuevos. En este grupo, $8 \%$ presentó multirresistencia y $19.3 \%$ resistencia global. La proporción de tuberculosis extremadamente resistente en los casos nuevos evaluados fue de $9 \% .{ }^{10}$ En un estudio realizado en Atehortúa por Muñoz SL y su equipo en 83 niños < 15 años con TB pulmonar, 1.22\% de los casos mostró resistencia a la rifampicina, utilizando la prueba molecular Xpert MTB/RIF. ${ }^{14}$

La información sobre los determinantes de la enfermedad en la edad pediátrica es escasa. Bezerra Souza y colegas reportaron coinfección TB-VIH en $4.5 \%(7 / 156)$ casos de TB en $<18$ años. ${ }^{6}$ Neyro $E$ y colaboradores en 92 casos pediátricos con TB ganglionar identificaron que $58.7 \%$ vivía en hacinamiento, $6.5 \%$ presentaba desnutrición, $4.4 \%$ enfermedad oncológica y $2.2 \%$ enfermedad reumatológica. ${ }^{7}$ En Paraguay, en 98 pacientes hospitalizados, $34.6 \%$ de ellos < 19 años hospitalizados presentaba coinfección TB-VIH y $23.4 \%$ (23/98) desnutrición. ${ }^{9}$

Datos de la OPS muestran que en 2016, 79\% de los $\leq 15$ años terminó con éxito el tratamiento, de los cuales $0.3 \%$ resultó en fracasos de tratamiento y $3 \%$ murieron por TB. ${ }^{15}$ Un punto fundamental en el abordaje y tratamiento de la TB en el niño lo constituyen los factores asociados tanto al abandono de la medicación como al óbito.

Así, Souza Viana PV y colaboradores refieren que en población indígena, los casos de retratamiento $(\mathrm{OR}=2.4 ; \mathrm{IC} 95 \%$ : 2.08-8.55) y con coinfección TB-VIH (OR = 8.2; IC95\%: 2.2-30.9) se asociaron al abandono del tratamiento y aquellos pacientes con TB extrapulmonar (OR = 1.8; IC95\%: 1.1-3.3), la edad < cuatro años (OR $=3.1$; IC95\%: $1.5-6.4)$ y los casos procedentes de las regiones del norte $(\mathrm{OR}=$ 2.8; IC95\%: 1.1-7.1) y centro-oeste del Brasil (OR = 2.8; IC95\%: 1.1-7.0) se asociaron al óbito (15). Por otro lado, en Paraguay la mortalidad en 98 pacientes $\leq 19$ años se asoció a la residencia de los pacientes en poblaciones con $<100,000$ habitantes (33.3 vs. $8.9 \%, p=0.02$. $R R=2.3$. IC 1.1-5.0) y a la forma de TB meníngea (46.2 vs. $15.3 \%, p<0.01$. $R R=3.0$; IC 1.4-6.5). ${ }^{16,17}$

La OMS recomienda la terapia preventiva de la TB en personas con $\mathrm{VIH}$ y todos los contactos convivientes de pacientes con TB. En ese sentido, el número de niños menores de cinco años que comenzaron el tratamiento preventivo contra la tuberculosis llegó a 349,000 en 2018, un aumento de cuatro veces desde 2015 , pero mucho menos que los cuatro millones que se estima que son elegibles.

Finalmente, es importante resaltar que en el año 2018, 4.8 millones de niños menores de cinco años sufrían de retraso en el crecimiento y 0.7 millones sufría de emaciación en América Latina y el Caribe. A ello se suma que en la edad pediátrica existe una brecha diagnóstica estimada en $69 \%$ de los casos, que uno de cada dos niños pierden acceso a la calidad de la atención de la TB y que ésta sólo la recibe uno de cada cuatro niños menores de cinco años que son elegibles para el tratamiento de TB preventiva, lo que pone de manifiesto que el abordaje de la TB en el niño debe ser integral y diferenciado, y que deben implementarse estrategias de manera urgente tanto para el abordaje de la TB como para el abordaje de los determinantes de la enfermedad.

\section{DIAGNÓSTICO}

El diagnóstico de la TB infantil es un reto para el clínico debido a la baja especificidad de los síntomas y a la baja rentabilidad de los métodos diagnósticos bacterianos. Es importante investigar la historia de contacto con pacientes bacilíferos en los últimos dos años, ya que el riesgo de desarrollar la enfermedad es mayor en este periodo. La edad es otro factor de riesgo, a menor edad mayor es el riesgo de sufrir enfermedad y de que ésta se disemine, presentando formas graves como la meningitis y TB miliar. ${ }^{15,18,19}$ La identificación de los signos y síntomas de la enfermedad pone a prueba la pericia del clínico, la TB se presenta con datos clínicos inespecíficos como pérdida o no ganancia de peso, fiebre prolongada, tos, adenopatías, diarrea prolongada, deformidad ósea, entre otros síntomas de enfermedad crónica. Otro aspecto a tomar en consideración es el estado inmunológico del paciente (condición de VIH, desnutrición, enfermedades inmunológicas). 
Rev Latin Infect Pediatr 2020; 33 (2): 66-73

Entre las pruebas diagnósticas de laboratorio se encuentran la prueba intradérmica de tuberculina (PPD) y las pruebas de interferón gamma liberado (siglas en inglés IGRAs). Las IGRAs son pruebas basadas en la producción de interferón gamma por las células $T$ sensibilizadas frente a antígenos de M. Tuberculosis, en la actualidad el más usado es el Quantiferon TB Gold, con sensibilidad similar a la tuberculina, pero con mayor especificidad, que no reacciona frente a micobacterias atípicas e inducidas por BCG.

Ambas pruebas ayudan a identificar si el paciente se ha expuesto al bacilo, pero no establecen diferencia entre infección y enfermedad. En pediatría son útiles para la identificación de tuberculosis latente y para tomar las medidas pertinentes. El punto de corte de PPD en la mayoría de los países se mantiene en $>5 \mathrm{~mm}$ para inmunosuprimidos $\mathrm{y}>10 \mathrm{~mm}$ en inmunocompetentes. ${ }^{20}$

Las imágenes diagnósticas (radiografía de tórax, ecografía y TAC) no son específicas para tuberculosis y se puede observar el complejo primario o complejo de Ghon (lesión pulmonar, linfadenitis y adenopatía hiliar), imágenes consolidadas, intersticiales, destructivas y cavitadas, además de derrame pleural. Hay que recordar que una radiografía de tórax normal no descarta enfermedad por tuberculosis. El ultrasonido y la tomografía se utilizan para localizar las lesiones, ver su extensión y tomar muestras. ${ }^{21}$

La bacteriología es la prueba estándar para el diagnóstico de la tuberculosis. El rendimiento de la bacteriología depende de la calidad de la muestra y concentración bacilar, siendo estos aspectos una limitante en pediatría, ya que el niño es paucibacilar y la muestra no siempre puede ser tomada por esputo espontáneo, teniendo que recurrir a esputo inducido y lavado gástrico (ambos con igual rendimiento) y aspirado nasofaríngeo. ${ }^{19}$

La baciloscopia se ha usado durante muchos años para el diagnóstico de la TB en niños, con un rendimiento no superior a $30 \%$ aun en muestras tomadas por broncoscopia. ${ }^{19}$ La identificación bacteriológica del Mycobacterium tuberculosis (MTB) se realiza con pruebas fenotípicas y genotípicas. Las pruebas fenotípicas permiten la identificación del MTB por cultivos, se puede realizar en medios sólidos (Lowenstein-Jensen) y en medios líquidos (BATER, MGIT 960). ${ }^{20}$

El cultivo en TBI nos permite confirmar diagnóstico cuando las pruebas moleculares son negativas y se tiene fuerte sospecha diagnóstica, además de ampliar la investigación de resistencia a fármacos antituberculosis.

Las pruebas genotípicas además de identificar el MTB permiten detectar mutaciones específicas del ADN del genoma del MTB que están asociadas a la resistencia del fármaco investigado. La OMS aprueba su uso a partir de 2008, siendo recomendada como prueba diagnóstica en niños a partir de 2010. Las pruebas genómicas más utilizadas son el GeneXpert MTB/Rif, GeneXpert MTB/Rif Ultra y Lipa (Line Probe Assays) y LAM (antígeno lipoarabinomanano)..$^{21-23}$ Estas pruebas permiten identificar el genoma del bacilo y la presencia de resistencia a fármacos de primera línea como la rifampicina e isoniazida, pilares del tratamiento y otros fármacos de segunda línea. Estas pruebas son de alta sensibilidad y especificidad, con rapidez de sus resultados y las pocas medidas de bioseguridad que deben tener los laboratorios que las procesan han permitido ofrecer tratamiento oportuno a los pacientes.

En 2010 la OMS aprueba el GeneXpert MTB/Rif como prueba inicial para los menores de 15 años con sospecha de tuberculosis, reiterando sus indicaciones en las directrices de 2020. ${ }^{23}$ Estas pruebas pueden realizarse en esputo, fluidos y tejidos. En recopilación realizada por la OMS sobre aportes del Xpert MTB/Rif se encontró una sensibilidad de 96-97\% y una especificidad de $99 \%$ para el diagnóstico de TB y resistencia a rifampicina. El Xpert MTB/ Rif Ultra mostró similar eficiencia. ${ }^{23}$

En estudio analizado en las directrices de OPS 2020 se observó la comparación de las pruebas moleculares (GeneXpert MTB/Rif y GeneXpert MTB/ Rif Ultra) en esputo, heces, nasofaringe y secreciones gástricas, encontrando que la sensibilidad varía con el tipo de muestra, (nasofaríngea $46 \%$, heces $61 \%$, esputo $65 \%$ y contenido gástrico $73 \%$ ) y la especificidad fue de 98 a $100 \%$ en todas las muestras. En cuanto a la determinación de resistencia a rifampicina, la sensibilidad fue de $90 \%$ y la especificidad de $98 \%$ en todas las muestras. ${ }^{24}$

Otros de los métodos moleculares es el LIPA (Line Probe Assays), que permite identificar mutaciones para el gen de la rifampicina (Gen rpoB) con una sensibilidad de $99 \%$ y especificidad $97 \%$ e isoniazida (katG) con sensibilidad de $61 \%$ y especificidad de $100 \%$, además de mutaciones para drogas de segunda línea como las quinolonas e inyectables con muestras directas e indirectas. ${ }^{22}$

La prueba TB LAM Ag ofrece detectar el antígeno LAM (lipoarabinomanano) en muestras de orina para 
Rev Latin Infect Pediatr 2020; 33 (2): 66-73

ayudar al diagnóstico de TB en personas $\mathrm{VIH}(+)$. Su sensibilidad global en población $\mathrm{VIH}(+)$ es de 21 a $54 \%$, pero aumenta a $56 \%$ si el recuento de CD4 es $<100$, con una especificidad de $95 \%$. Esta prueba no se recomienda de forma rutinaria, sólo en personas con infección por VIH con recuentos de CD4 bajos o que estén gravemente enfermos. ${ }^{22}$

En las directrices la OMS 2020 recomienda el uso de pruebas moleculares (GeneXpert MTB/Rif y Xpert Ultra) en la evaluación diagnostica niños con sospecha de TB pulmonar y extrapulmonar, TB-MDR o TB/ HIV en muestras de esputo, secreciones gástricas, nasofaríngea y heces. ${ }^{23}$

En estos momentos contamos con herramientas bacteriologías moleculares de alto rendimiento para el diagnóstico de la TB en niños, debemos mejorar la calidad de la muestra para garantizar su rendimiento. La posibilidad de que las muestras de orina y heces tengan buen rendimiento abre las puertas a mejores diagnósticos en pediatría. Por el momento, el diagnóstico de TB en la población infantil tiene un componente clínico y epidemiológico importante.

\section{TRATAMIENTO}

El enfoque actual para tratar la TB infantil se deriva de datos extrapolados de ensayos clínicos en adultos; sin embargo, el espectro de la enfermedad infantil y la tasa de progresión de la enfermedad varía significativamente entre los rangos de edad. ${ }^{25}$ Los niños pequeños (< dos años de edad) tienen más probabilidades de desarrollar enfermedad diseminada (miliar) y meningitis tuberculosa, mientras que los adolescentes (> 10-18 años) tienden a desarrollar enfermedad cavitaria de tipo adulto. ${ }^{26}$ En general, los niños tienden a desarrollar enfermedad paucibacilar con un espectro heterogéneo de enfermedad clínica.

Los objetivos de la terapia de TB son: 1) reducir el riesgo de muerte y discapacidad, 2) reducir la transmisión continua de $M$. tuberculosis, 3) asegurar una cura duradera sin recaída, y 4) prevenir el desarrollo de resistencia a los medicamentos durante la terapia. M. tuberculosis es difícil de tratar debido a su lenta tasa de división (hasta 24 horas), su actividad metabólica variable con bacilos inactivos, o intermitentemente con activos que lo hacen tolerante a muchos medicamentos y su ubicación en varios compartimentos corporales que afectan la penetración del medicamento y actividad.

El tratamiento de la tuberculosis requiere la administración de múltiples medicamentos durante varios meses. La participación del niño y sus padres es crucial, con una explicación de la decisión de iniciar el tratamiento, los medicamentos elegidos, la prevención de los efectos secundarios y la importancia de la terapia directamente observada (TDO). LA TDO permite una mejor adherencia al tratamiento y por ende, un mayor porcentaje de curación de los pacientes con disminución de la probabilidad de recaídas y por tanto, menor mortalidad. ${ }^{27}$

La OMS recomienda la selección de un régimen de tres a cuatro fármacos en función de: 1) la ubicación de la tuberculosis, 2) el riesgo de resistencia a la isoniazida y 3) la prevalencia del $\mathrm{VIH} .{ }^{28,29}$ Según la OMS, los niños con sospecha o confirmación de tuberculosis pulmonar o linfadenitis tuberculosa periférica que vive en entornos con baja prevalencia de $\mathrm{VIH}$ o baja resistencia a la isoniazida y los niños que son $\mathrm{VIH}$ negativos pueden tratarse con un régimen de tres fármacos ( $\mathrm{HRZ}$ ) durante dos meses seguido de un régimen de dos fármacos $(\mathrm{HR})$ durante cuatro meses. ${ }^{30}$

Niños que viven en entornos donde la prevalencia del VIH es alta o donde la resistencia a la isoniazida es alta, o ambas, con sospecha o confirmación de tuberculosis pulmonar o linfadenitis periférica, o los niños con enfermedad pulmonar extensa que viven en entornos de baja prevalencia del VIH o baja resistencia a la isoniazida, deben ser tratados con un régimen de cuatro medicamentos durante dos meses seguido de un régimen de dos medicamentos durante cuatro meses. ${ }^{31}$

Con el fin de lograr concentraciones apropiadas de medicamentos en niños pequeños y teniendo en cuenta la evidencia farmacocinética, las dosis recomendadas de estos medicamentos antituberculosos de primera línea fueron revisados por la OMS en 2010. Los niños metabolizan los medicamentos más rápidamente que los adultos, lo que resulta en una concentración sérica más baja. La OMS recomienda las siguientes dosis de medicamentos antituberculosos para el tratamiento de la tuberculosis en niños: ${ }^{22-36}$

a) Isoniazida $(\mathrm{H})-10 \mathrm{mg} / \mathrm{kg}$ (rango $10-15 \mathrm{mg} / \mathrm{kg}$ ); dosis máxima 300 mg/día;

b) Rifampicina (R) - $15 \mathrm{mg} / \mathrm{kg}$ (rango $10-20 \mathrm{mg} / \mathrm{kg}$ ); dosis máxima 600 mg/día;

c) Pirazinamida (Z) - $35 \mathrm{mg} / \mathrm{kg}(30-40 \mathrm{mg} / \mathrm{kg})$.

d) Etambutol (E) - $20 \mathrm{mg} / \mathrm{kg}(15-25 \mathrm{mg} / \mathrm{kg})$

Ya que la prevalencia y el riesgo de tuberculosis farmacorresistente pueden ser difíciles de 
determinar, la AAP y la mayoría de los expertos incluyen etambutol (EMB) como parte del régimen de fase intensiva para niños con tuberculosis. La AAP recomienda el régimen de cuatro fármacos (HRZE) durante dos meses seguido de un régimen de dos fármacos (HR) durante cuatro meses para la tuberculosis pulmonar sospechada o confirmada. ${ }^{33,37}$

Adicionalmente, durante la fase de continuación del tratamiento se pueden considerar regímenes de tres veces por semana para los niños no infectados por el VIH y que viven en entornos con una terapia bien establecida y observada directamente (DOT). EI más efectivo de estos métodos hasta la fecha es la administración de dosis de tratamiento intermitente de medicamentos para la tuberculosis (una o dos veces por semana) a través de DOT. ${ }^{30-32}$ Aunque el DOT puede no estar disponible para todos los niños en todos los entornos, se ha demostrado que aumenta la adherencia en niños con infección de TB, independientemente del nivel socioeconómico, el estado de inmigración y la preferencia de idioma. ${ }^{38}$

Por otro lado, otra de las recomendaciones que enfatiza la OMS, es que la estreptomicina no debe usarse como parte de los regímenes de tratamiento de primera línea para niños con tuberculosis pulmonar o linfadenitis periférica tuberculosa. ${ }^{33}$

Los niños con tuberculosis pulmonar comprobada que vivan en una zona de alta endemicidad de TB-MDR pueden ser tratados con fluoroquinolona. La decisión debe ser tomada por un médico con experiencia en el manejo de la tuberculosis pediátrica. ${ }^{39,40}$

Los niños que reciben tratamiento para la infección de TB deben revisarse periódicamente para evaluar la adherencia, abordar las preocupaciones familiares con la terapia, corregir la importancia de continuar la terapia y evaluar los posibles efectos secundarios.

La evaluación de laboratorio basal y en serie no es necesaria para niños previamente sanos que reciben tratamiento para la infección de TB. Sin embargo, un niño que recibe otros medicamentos que pueden causar hepatotoxicidad o que tiene una enfermedad hepática existente debe tener una monitorización de laboratorio basal y en serie de las enzimas hepáticas séricas. Alrededor de 3\% de los niños que reciben medicamentos para la TB tienen una elevación asintomática transitoria de las transaminasas hepáticas (de dos a tres veces el límite superior de lo normal) y no requieren una modificación del tratamiento. ${ }^{41}$ Todo niño con signos o síntomas de hepatotoxicidad o elevación de la enzima hepática por encima de cinco veces el límite superior a la normalidad debe suspender el tratamiento de la tuberculosis y ser evaluado médicamente..$^{42}$

\section{SUPLEMENTACIÓN CON PIRIDOXINA}

La isoniazida interfiere con el metabolismo de la piridoxina (B6), lo que conduce a una deficiencia dependiente de la dosis de vitamina B6 biológicamente activa. ${ }^{43.44}$ La deficiencia de piridoxina conduce a una neuropatía periférica sensoriomotora mixta que se manifiesta como hormigueo, entumecimiento o debilidad del pie. Estos síntomas son reversibles con suplementos de piridoxina.

La deficiencia sintomática de piridoxina es rara en niños, ${ }^{45,46}$ lo que se cree que se asocia en parte al metabolismo más rápido de $\mathrm{INH}$ en niños. ${ }^{46}$ Los niños que están desnutridos, amamantados exclusivamente, infectados por el VIH, adolescentes con dietas deficientes en proteínas o que están embarazadas, y los niños que reciben altas dosis de INH tienen mayor riesgo de desarrollar deficiencia sintomática de piridoxina. Para estos niños se recomienda la suplementación con piridoxina $1-2 \mathrm{mg} / \mathrm{kg} /$ día (rango habitual $10-100 \mathrm{mg} /$ día) para prevenir los síntomas. La deficiencia aguda de piridoxina después de una sobredosis de isoniazida puede causar convulsiones tónico-clónicas generalizadas que son difíciles de controlar hasta que se corrija la deficiencia. ${ }^{46}$

\section{PRONÓSTICO}

La resistencia a isoniazida tiene un impacto negativo en el pronóstico de los pacientes. De acuerdo con Gegia M y colaboradores ${ }^{17}$ la presencia de monorresistencia a isoniazida representa una falla terapéutica de 11\% (IC 95\%, 6-17) en comparación con $2 \%$ (IC 95\%, 1-3) en casos de susceptibilidad. La recurrencia ocurre en 10\% (IC 95\%, 5-15) de casos. La combinación de falla terapéutica, recurrencia o ambos ocurre en $15 \%$ de casos con INH-R, sin diferencia significativa en relación al esquema terapéutico implementado. La adquisición de genes de resistencia se documentó en $6 \%$ de los casos $\mathrm{INH}-\mathrm{R}$, que fue 10 veces mayor que la adquisición de resistencia en casos sensibles a INH. De los casos que adquirieron genes de resistencia para otras drogas anti-TB, 96\% (IC 95\%, 93-99) culminó en cepas multidrogorresistentes. 


\section{CONCLUSIÓN}

El abordaje de la TB no requiere de una gran infraestructura hospitalaria, sino de pediatras, médicos de familia y personal de salud entrenado para la sospecha diagnóstica precoz y tratamiento oportuno en el niño. Además, se requiere de una red de diagnóstico laboratorial, $\mathrm{RRHH}$ responsables de TB en todos los establecimientos de salud, medicamentos con formulaciones pediátricas, seguimiento del tratamiento y abordaje de los determinantes de la enfermedad. La brecha diagnóstica en TB infantil a nivel de las Américas, el bajo éxito de tratamiento observado, altas tasas de abandono y mortalidad ponen de manifiesto la debilidad de los sistemas de salud de los países de la región, la falta de aplicación de normas de TB para el abordaje de la TB latente y la falta de entrenamiento de los pediatras para abordar esta patología.

La tuberculosis infantil se mantiene como un reto para la salud pública mundial. Es obligatorio disponer de información epidemiológica y terapéutica actualizada en niños para facilitar las acciones del personal de salud. Por otro lado, se requiere de un fuerte compromiso de las autoridades de salud de los países y otros sectores gubernamentales y no gubernamentales para la óptima implementación de la estrategia de la OMS «Fin de la TB», cuyas metas en 2035 son: reducir en $95 \%$ el número de muertes por TB y en $90 \%$ la incidencia en relación al año 2015, consiguiendo que ninguna familia tenga que hacer frente a gastos catastróficos debido a la tuberculosis.

\section{REFERENCIAS}

1. World Health Organization. Global Tuberculosis Report 2019. Geneva, Switzerland: World Health Organization; 2019.

2. World Health Organization. Tuberculosis. Disponible en: http://www.who.int/mediacentre/factsheets/fs104/en/.

3. World Health Organization. Global Tuberculosis Report 2016. Disponible en: http://www.who.int/tb/publications/ global_report/en/.

4. Seddon JA, Jenkins HE, Liu L, Cohen T, Black RE, Vos T et al. Counting children with tuberculosis: why numbers matter. Int J Tuberc Lung Dis. 2015; 19 Suppl 1 (0 1): 9-16.

5. Comité Nacional de Neumonología; Comité Nacional de Infectología. Criterios de diagnóstico y tratamiento de la tuberculosis infantil. Resumen ejecutivo. Arch Argent Pediatr. 2016; 114 (2): 189-190.

6. Mohapatra PR, Janmeja AK. Tuberculous lymphadenitis. J Assoc Physicians India. 2009; 57: 585-590.

7. Sousa GJB, Silva JCO, Queiroz TV, Bravo LG, Brito GCB, Pereira AS et al. Clinical and epidemiological features of tuberculosis in children and adolescents. Rev Bras Enferm. 2019; 72 (5): 1271-1278.
8. Neyro SE, Squassi IR, Medín M, Caratozzolo A, Martínez BA, Cerqueiro MC. Tuberculosis ganglionar periférica en pediatría: 16 años de experiencia en un centro pediátrico de tercer nivel de Buenos Aires, Argentina. Arch Argent Pediatr. 2018; 116 (6): 430-436.

9. Carreto-Binaghi L, Juárez E, Guzmán-Beltrán S, Herrera M, Torres M, Sarabia C et al. Diagnosis of childhood tuberculosis at INER in 2015-2017. Neumol Cir Torax. 2018; 77 (4): 258-266.

10. Martínez de Cuellar C, Lovera D, Gatti L, Ojeda L, Apodaca $\mathrm{S}$, Zarate $\mathrm{C}$ et al. Tuberculosis: Factores de riesgo asociados a mortalidad en pacientes $\leq 19$ años hospitalizados en el Instituto de Medicina Tropical. Pediatr (Asunción). 2019; 46 (2): 77-81.

11. Zabaleta A, Llerena C, Valbuena A. Vigilancia por el laboratorio de la tuberculosis resistente en menores de 15 años, Colombia, 2010 a 2015. Biomédica. 2019; 39 (2): 330338.

12. Vázquez RJ , Acosta GC, Miranda NM, Fuentes PY, Labra ZM, Pacheco RD, Solórzano SF. A case-series analysis of tuberculosis in pediatric patients treated in a tertiary level hospital. Bol Med Hosp Infant Mex. 2017; 74 (1): 27-33.

13. Sreeramareddy CT, Panduru KV, Verma SC, Joshi HS, Bates MN. Comparison of pulmonary and extrapulmonary tuberculosis in Nepal-a hospital-based retrospective study. BMC Infect Dis. 2008; 8: 8.

14. Marais BJ, Graham SN, Cotton MF, Beyers N. Diagnostic and management challenges for childhood tuberculosis in the era of HIV. J Infect Dis. 2007; 196 Suppl 1: S76-85.

15. Gegia M, Winters N, Benedetti A, van Soolingen D, Menzies D. Treatment of isoniazid-resistant tuberculosis with first-line drugs: a systematic review and meta-analysis. Lancet Infect Dis. 2017; 17 (2): 223-234.

16. Atehortúa MS, Rendón MJ, Cárdenas MS, Arango FC, Cornejo OJ. Xpert MTB/RIF( $r$ ) as a diagnostic tool in a cohort of children under 15 years of age with clinical suspicion of pulmonary tuberculosis in a hospital of high complexity in Medellin. Infect. 2017; 21 (1): 25-31.

17. De Sousa VP, Barbosa CS, Bierrenbach A, Basta P. Tuberculosis entre niños y adolescentes indígenas en Brasil: factores asociados al óbito y al abandono del tratamiento. Cad. Saúde Pública. 2019; 35 (Suppl. 3): e00074218.

18. Stagg HR, Harris RJ, Hatherell HA, Obach D, Zhao H, Tsuchiya $\mathrm{N}$ et al. What are the most efficacious treatment regimens for isoniazid-resistant tuberculosis? A systematic review and network meta-analysis. Thorax. 2016; 71 (10): 940-949.

19. Ramos AJ. Actualización en tuberculosis infantil. EEPap. 2018; 2253-2257.

20. Farga V, Caminero J. Tuberculosis. 3ra ed. Santiago de Chile: Mediterráneo; 2011.

21. Guarda ME . Tuberculosis en el niño ¿Cómo se diagnostica? Revista Médica Clínica Las Condes. 2017; 28 (1): 104-110.

22. Arias M, Herrera T. Nuevos métodos para el diagnóstico de la tuberculosis. Rev Chil enferm resp. 2016; 32 (4): 254-259.

23. Herrera FA. Nuevos métodos para el diagnóstico de la tuberculosis. Rev Chil Enferm Respir. 2016; 32: 254-259.

24. OPS. Rapid Communication: Molecular assays as initial tests for the diagnosis of tuberculosis and rifampicin result. Washington: OPS, 2020.

25. World Health Organization. Global tuberculosis report. World Health Organization, Geneva, Switzerland. 2017. Available at: http://www.who.int/tb/publications/global_report/en/. 
Rev Latin Infect Pediatr 2020; 33 (2): 66-73

26. Marais BJ, Gie RP, Schaaf HS, Hesseling AC, Obihara $\mathrm{CC}$, Starke JJ et al. The natural history of childhood intrathoracic tuberculosis: a critical review of literature from the pre-chemotherapy era. Int J Tuberc Lung Dis. 2004; 8 (4): 392-402.

27. Khan A, Sterling TR, Reves R, Vernon A, Horsburgh CR. Lack of weight gain and relapse risk in a large tuberculosis treatment trial. Am J Respir Crit Care Med. 2006; 174: 344-348.

28. World Health Organization. Rapid advice: treatment of tuber- culosis in children. Geneva: WHO; 2010.

29. World Health Organization. Guidance for national tuberculosis programmes on the management of tuberculosis in children. 2nd ed. Geneva, Switzerland: WHO; 2014

30. Zar HJ, Hanslo D, Apolles P, Swingler G, Hussey G. Induced sputum versus gastric lavage for microbiological confirmation of pulmonary tuberculosis in infants and young children: a prospective study. Lancet. 2005; 365: 130-134.

31. Santos G, Oliveira O, Gaio R, Duarte R. Effect of isoniazid resistance on the tuberculosis treatment outcome. Arch Bron Coneumol. 2017. pii: S0300-2896(17)30215-6.

32. American Academy of Pediatrics, Committee on Infectious Dis- eases. 2015 Red Book: Report of the Committee on Infectious Diseases. 30th ed. Elk Grove Village, IL: AAP; 2015.

33. Cruz AT, Starke JR. Twice-weekly therapy for children with tuberculosis infection or exposure. Int J Tuberc Lung Dis. 2013; 17: 169-174.

34. Parsyan AE, Saukkonen J, Barry MA, Sharnprapai S, Horsburgh CR. Predictors of failure to complete therapy for latent tuberculosis infection. J Infect. 2007; 54 (3): 262-266.

35. Kwara A, Herold JS, Machan JT, Carter EJ. Factors associated with failure to complete isoniazid treatment for latent tuberculosis infection in Rhode Island. Chest. 2008; 133 (4): 862-868.

36. Harausz EP, Garcia-Prats AJ, Law S, Schaaf HS, Kredo $\mathrm{T}$, Seddon $\mathrm{J}$ et al. Treatment and outcomes in children with multidrug-resistant tuberculosis: a systematic review and individual patient data meta-analysis. PLoS Med. 2018; 15 (7): e1002591.

37. The Sentinel Project for Pediatric Drug-Resistant Tuberculosis. Management of multidrug-resistant tuberculosis in children: a field guide: Boston, USA; 2019
[Access Year |;Access Date; 2019; 10 April]. Fourth: [Available from: http://sentinel-project.org/wp-content/ uploads/2019/02/Updated_DRTB-Field-Guide-2019-V3. pdf.

38. O'Brien RJ, Long MW, Cross FS, Lyle MA, Snider DE. Hepatotoxicity from isoniazid and rifampin among children treated for tuberculosis. Pediatrics. 1983; 72 (4): 491-499.

39. Palusci VJ, O'Hare D, Lawrence RM. Hepatotoxicity and transaminase measurement during isoniazid chemoprophylaxis in children. Pediatr Infect Dis J. 1995; 14: 144-148.

40. Biehl JP, Vilter RW. Effects of isoniazid on pyridoxine metabolism. JAMA. 1954; 156: 1549-1552.

41. Biehl JP, Vilter RW. Effect on isoniazid on vitamin B6 metabolism: its possible significance in producing isoniazid neuropathy. Proc Soc Biol Med. 1954; 85: 389-392.

42. Pellock JM , Howell J, Kendig EL Jr, Baker H. Pyridoxine deficiency in children treated with isoniazid. Chest. 1985; 87 (5): 658-661.

43. Morales SM, Lincoln EM. The effect of isoniazid therapy on pyridoxine. Rev Tuberc. 1957; 75: 594-600.

44. Cillers K, Labadarios D, Schaaf HS et al. Pyridoxal-5phosphate plasma concentrations in children receiving tuberculosis chemotherapy including isoniazid. Acta Paediatr. 2010; 99: 705-710.

45. Schaaf HS, Parkin DP, Seifart HI, Werely CJ, Hesseling PB, van Helden PD et al. Isoniazid pharmacokinetics in children treated for respiratory tuberculosis. Arch Dis Child. 2005; 90 (6): 614-618.

46. Minns AB, Ghafouri N, Clark RF. Isoniazid induced status epilepticus in a pediatric patient after inadequate pyridoxine therapy. Pediatr Emerg Care. 2010; 26 (5): 380-381.

\section{Correspondencia:}

José Iván Castillo Bejarano

Departamento de Pediatría, División de Infectología Pediátrica, Hospital Universitario «Dr. José Eleuterio González», Monterrey, Nuevo León

Avenida Madero y Gonzalitos S/N,

64460, Monterrey, Nuevo León, México,

Tel: (81) 83-89-11-11

E-mail: jicastillobejarano@gmail.com 